\section{Contrast Extravasation Predicts Hematoma Growth: Where to Now?}

The findings of Kim et $\mathrm{al}^{1}$ regarding CT angiography and outcome after intracerebral hemorrhage (ICH) are of importance and complement our previously published work demonstrating an independent association between extravasation, coined the "CT angiogram (CTA) spot sign" and hematoma growth. ${ }^{2}$ Crucially the study by Kim et al ${ }^{1}$ also demonstrated an independent association with clinical outcome. A prospective validation study of the CTA spot sign has recently shown a similar result. ${ }^{3}$ To strengthen current literature, it would be helpful for the authors to provide their working definition of hematoma growth and indicate the diagnostic performance of extravasation for hematoma growth. The number of patients in each group undergoing hematoma growth is also important. The finding of normal CTA with postcontrast CT is significant. How do the authors account for these differences? Did any of these patients demonstrate hematoma expansion? Was there any association with increased international normalized ratio?

In the present study, the mean time to presentation was 10 hours, with a reported incidence of extravasation of $19 \%-23 \%$. The incidence of the spot sign in our study where patients were scanned within 3 hours was $33 \%$. This suggests a reduction in the risk of extravasation with time. We have demonstrated previously that the specificity of extravasation for hematoma growth is reduced with increasing time to scan, ${ }^{4}$ probably due to nonspecific secondary vascular injury caused by hematoma shearing. These 2 observations argue against an extended time window for intervention, as suggested by the authors.

This study and others provide early evidence of a potentially potent sign that could accurately identify patients at risk of significant hematoma growth. These patients would be ideal candidates for randomized treatment trials of early hemostatic therapy. Two complementary studies (the Spot Sign for Predicting and Treating ICH
Growth Study and the Spot Sign Selection of ICH to Guide Hemostatic Therapy Study) are being planned to prospectively evaluate the CTA spot sign for the prediction of hematoma growth. These studies will assess the feasibility of hyperacute CTA investigation and the reliability of spot sign identification in an emergency setting and will provide pilot clinical data by randomly assigning patients with a spot sign to treatment with activated recombinant factor VII or placebo. The exciting findings of Kim et $\mathrm{al}^{1}$ further emphasize the need for such prospective studies.

\section{References}

1. Kim J, Smith A, Hemphill JC 3rd, et al. Contrast extravasation on CT predicts mortality in primary intracerebral hemorrhage. AJNR Am J Neuroradiol 2008;29:520-25

2. Wada R, Aviv RI, Fox AJ, et al. CT Angiography “spot sign” predicts hematoma expansion in acute intracerebral hemorrhage. Stroke 2007;38:1257-62

3. Demchuk AM KJ, Tymchuk S, O'Reilly C, et al for the PREDICT/Sunnybrook ICH CTA Study Group. Multicentre prospective study demonstrates feasibility of CT-angiography in intracerebral hemorrhage and validity of "spot sign" for hematoma expansion prediction. International Stroke Conference. New Orleans: February 20-22, 2008.

4. Goldstein JN, Fazen LE, Snider R, et al. Contrast extravasation on CT angiography predicts hematoma expansion in intracerebral hemorrhage. Neurology 2007;68:889-94

R.I. Aviv

D. Gladstone

J. Goldstein

M. Flaherty

J. Broderick

A. Demchuk

for the Spot Sign for Predicting and Treating ICH Growth and Spot Sign Selection of ICH to Guide Hemostatic Therapy Investigators Sunnybrook Health Science Centre Toronto, Ontario, Canada

DOI 10.3174/ajnr.A1140 